\title{
Suitability of Fast Healthcare Interoperability Resources (FHIR) for Wellness Data
}

\author{
Sarita Pais \\ AUT, Whitireia Polytechnic \\ Sarita.pais@whitireia.ac.nz
}

\author{
Dave Parry \\ AUT \\ Dave.parry@aut.ac.nz
}

\author{
Yungfeng Huang \\ Whitireia Polytechnic \\ yungfeng@whitireianz.ac.nz
}

\begin{abstract}
Wellness data generated by patients using smart phones and portable devices can be a key part of Personal Health Record (PHR) data and offers healthcare service providers (healthcare providers) patient health information on a daily basis. Prior research has identified the potential for improved communication between healthcare provider and patient. However the practice of sharing patient generated wellness data has not been widely adopted by the healthcare sector; one of the reasons being the lack of interoperability preventing successful integration of such device generated data into the PHR and Electronic Health Record (EHR) systems. To address the interoperability issue it is important to make sure that wellness data can be supported in healthcare information exchange standards. Fast Healthcare Interoperability Resources (FHIR) is used in the current research study to identify the technical feasibility for patient generated wellness data. FHIR is expected to be the future healthcare information exchange standard in the healthcare industry.

A conceptual data model of wellness data was developed for evaluation using FHIR standard. The conceptual data model contained blood glucose readings, blood pressure readings and Body Mass Index (BMI) data and could be extended to accept other types of wellness data. The wellness data model was packaged in an official FHIR resource called Observation. The research study proved the flexibility of adding new data elements related to wellness in Observation. It met the requirements in FHIR to include such data elements useful in self-management of chronic diseases. It also had the potential in sharing it with the healthcare provider system.
\end{abstract}

\section{Introduction}

A PHR contains information related to the care of a patient and is accessible by the patient and healthcare provider. PHR includes information from the
Electronic Health Record (EHR) such as patient's health conditions, medical history and laboratory test results which is shared with the patient.

Wellness data can be a key part among different parts of PHR data, especially as mobile and wearable device technology advances and can integrate with the PHR system.

Patient managed data from PHR can be submitted into their healthcare provider's EHR system, helping clinicians make better decisions. It also improves the sharing of medical records, increases patient safety and reduces healthcare costs [1]. Although patient managed wellness data and PHR have been discussed in research studies, its adoption in the real world is limited. There are several reasons for this such as interoperability issues, uncertainty of the quality of health data from patients, trustworthiness of wellness data from patients and possible information overload as patients generate too much data [2] [3]. This research study attempts to address the interoperability issues.

Integrating PHR data, especially patient generated wellness data into EHR will improve communication between patients and clinicians [4] and consequently help clinicians better understand the patient's health conditions. Several previous research studies have demonstrated that the collection and utilization of wellness data from patients have the potential for healthcare organizations to provide better health services [5]. Intervention studies have proved better quality of diabetes care for VA patients [6]. Most PHR systems are physician-oriented [7] and do not include functionalities for patients to upload their wellness data. However in the instance of telehealth care and management of chronic diseases there were significant improvements in health outcomes and satisfaction levels [8]. Similarly sharing wellness data from PHR has the potential to improve health outcomes. Among the issues preventing PHR and wellness data being widely accepted was the lack of interoperability with EHR systems. Interoperability will allow PHR and EHR systems from different vendors to communicate, 
exchange data, and use the wellness data that has been exchanged through healthcare information exchange standard.

Fast Healthcare Interoperability resources (FHIR) is a draft standard developed by Health Level Seven International (HL7), a not-for-profit organization dedicated to providing healthcare information exchange standards. FHIR is expected to be the next generation standard of healthcare information exchange and its support for wellness data and PHR is key to whether wellness data and PHR could be widely adopted in the healthcare industry. However the suitability of FHIR for wellness data has not yet been fully demonstrated. In 2015 when this research project was initiated there were 93 FHIR resources published by HL7 [9]. These resources covered medication, diagnostics, device interactions and other clinical aspects such as administration and infrastructure. None of these resources are specially designed for wellness data or PHR. Hence this research attempts to evaluate the suitability of FHIR for wellness data.

This research project had one main research question:

What is the suitability of FHIR for patient managed wellness data?

This question was further sub-divided into:

- How can a model of patient managed wellness data be developed?

- How can the developed model of patient managed wellness data be implemented using FHIR?

- What are the issues and constraints expressing wellness data with FHIR?

To answer these research questions, a conceptual data model of wellness data was developed based on literature review about self-management of chronic diseases. Existing FHIR resources were reviewed to identify if they could be used to represent wellness data managed by patients. Official FHIR resources were used to upload and query data items included in the wellness data conceptual model to investigate the possibility of expressing wellness data with existing FHIR resources.

\section{Healthcare Information Exchange Standard}

Industry standards are essential for adopting new technologies to resolve interoperability issues of sharing wellness data from PHR, mobile apps and devices. HL7 Version 2 (V2) is a widely used healthcare information exchange standard. However it fails to integrate well with new web technologies such as Restful APIs. HL7 introduced FHIR with the best features of HL7 Versions 2 and 3. FHIR is now under a draft use version but it is widely expected to be the next generation of worldwide healthcare information exchange standard. The need for FHIR has risen as HL7 V3 is complex and takes longer for development; HL7 V2 is old style and is not compatible with new technologies such as mobile applications and cloud computing [10]. Most resources in FHIR are catered to clinical data in EHR system. The suitability of FHIR for wellness data is not known. This research was motivated by the need to evaluate the suitability of FHIR for wellness data.

\section{Identification of Chronic Diseases and Representing Wellness Data}

In order to investigate the suitability of FHIR for wellness data the first step was to identify representable data from a wide range of required wellness data and develop a conceptual data model of it, which could then be used to test with FHIR resources. Routinely generated wellness data and PHR were not considered important to be recorded in EHR systems. However there were limited studies mainly focused on chronic diseases and related data collection and utilization. Chronic diseases were identified as one of the biggest challenges to society and wellness data of patients with chronic diseases was useful to clinicians. The Institute of Medicine [11] in the United States declared chronic diseases a public health crisis and advocated comprehensive health monitoring systems that support living well as a major component of a solution to the problem. The same can be applied to any country with high rates of chronic diseases [12]. Diabetes [13] and hypertension [14] were significant chronic diseases and research relating to these provided a good basis for considering data elements for representing wellness data in this research project.

A large scale research program [8] called "Care Coordination / Home Telehealth" provided chronic disease care management for patients with diabetes, heart failure, depression and other chronic conditions common to older veterans. Biometric devices such as blood glucometers and blood pressure meters were used to collect vital signs and disease management data from patients. These data were then transferred to the server of healthcare providers in line with HL7 standards. The results over four years were a $25 \%$ reduction in bed days of care, a $19 \%$ reduction in hospital admissions and high levels of patient satisfaction.

In another research by Quinn, et al. [15], glucose readings and physical activity data generated by the meters were uploaded to the server where clinicians reviewed the data and gave feedback to the patients by 
email. The result showed significant improvement in Haemoglobin A1c (HbA1c) of $-2.03 \%$.

In another example of sharing PHR data into EHR, a middleware layer application server was set up based on ontology information model to map the data [16]. In this architecture a PHR system exports patient data into CCR/CCD data format and an EHR system imports data using HL7/CDA data format.

Publicly available application programming interfaces (APIs) have the potential to share patient generated health data from participating mobile apps and sensor devices to build a software ecosystem. Interoperability can be achieved through this approach. Individuals have access to their health and wellness data and can share it with clinicians. Such practices are recommended by researchers [17] [18]. FHIR currently does not include patient generated wellness data. If an official data schema for wellness data is available in FHIR then the mashed up data from mobile apps could easily be tethered into EHR through FHIR standard of exchange data. Most commercial mobile apps available on different platforms are standalone and not integrated for use in an ecosystem.

There has been research on developing new mobile apps for the collection and utilization of blood glucose readings in self-management [19] [20] [21] [22] [23] [24] and not necessarily for easy sharing of blood glucose readings. There were significant improvements in health outcomes in some but not all cases. Lee [25] reported a project where pulse data from digital pulse examinations of patients were integrated in an EHR system as part of remote medical services.

All the above research focused on projects of comprehensive study of wellness data collection and utilization. Several scenarios were proposed and discussed to illustrate how a patient could get their health data, upload it and get feedback from health professionals [26]. Blood glucose and blood pressure readings had been successfully collected and utilized in pilot projects. This made blood glucose and blood pressure a good choice to be included in the proposed wellness data model. BMI, a good indicator of chronic diseases, was also included in the model.

\section{Organization of Wellness Data Model}

To make the conceptual model of wellness data suit common practice in the healthcare industry, information on self-monitoring activities including test frequency, test conditions, different types of readings should also be taken into account.

With regard to the self-monitoring of blood glucose levels, one blood glucose reading could be very different from another depending on whether the reading was taken before or after a meal. When a blood glucose reading is recorded, the testing conditions should also be recorded. The testing frequency is also important. A testing frequency is based on the patient's type of diabetes. As a common suggestion for type 2 diabetes patients [27] a three point daily testing routine includes one fasting, one postprandial and one postabsorptive. For patient with type 1 diabetes, a four to eight point daily testing was recommended. Regardless of the testing regimen, patients were encouraged to collect data on blood glucose levels relative to meals [28]. The blood glucose readings were recorded and some comments on food consumed too were considered.

The test for blood pressure was relatively simple. As suggested by Imai et al. [29] blood pressure should be measured at least once in the morning and once in the evening for self-monitoring at home. Pickering [30] made the same suggestions.

\section{A Model of Patient Managed Wellness Data}

A conceptual model of wellness data was developed by identifying key data elements about chronic diseases that were required. Thus blood glucose, blood pressure readings and BMI data were chosen as representing wellness data. The developed wellness data model is shown in Figure 1. The data model included a reference to the patient to whom the wellness data belonged and recorded the time when data was collected. The multiple readings of blood glucose, blood pressure readings and BMI were organized in a list. Each blood glucose reading consisted of the type of test (fasting, before or after each meal), the test reading, the unit and the time of test. 


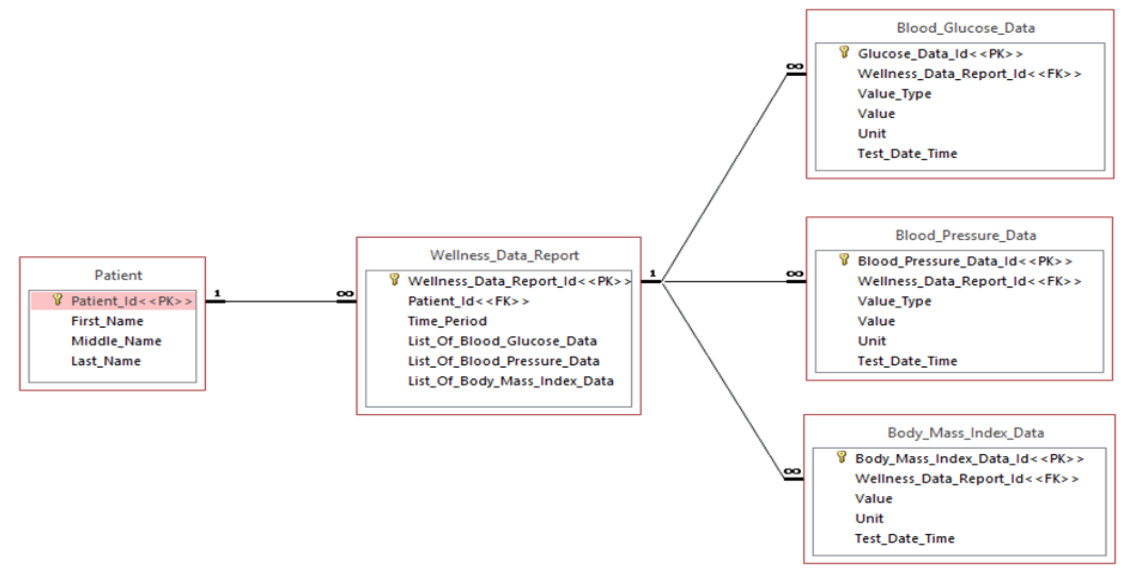

Figure: 1: Wellness data model

\section{Expressing Wellness Data with Existing Resources}

Although existing FHIR resources were not specially designed for wellness data and there was no clear demonstration of their support, it was possible to organize wellness data within "Observation" which is an existing official resource. Observation was used to record diagnosis, monitor progress, determine baselines and patterns and even capture demographic characteristics. An Observation record could not directly contain multiple values, so the developed wellness data model had to be divided into individual data items so as to be expressed within Observation. This also meant that the 'time-period' defined in the data model could now be explicitly included in each Observation resource of wellness data items. The different types of blood glucose and blood pressure readings were distinguished using existing clinical coding system LOINC [31]. These LOINC codes could distinguish whether the blood glucose test was done using a glucometer at home and whether it was a postprandial or fasting test. The LOINC code for a postprandial glucose reading using glucometer is 14770-2. The fasting blood glucose LOINC code is 41604-0. These coding enhance the semantic meaning of the data in a clinical environment. Blood pressure readings too have distinctive LOINC codes to differentiate systolic and diastolic readings. All data items identified in the wellness data model could be organized within the existing Observation resource.

A XML document containing blood glucose readings was successfully uploaded to the FHIR server within the Observation resource related to a patient ID. The uploaded blood glucose data could then be accessed with the Observation resource ID, as shown in Figure 2.

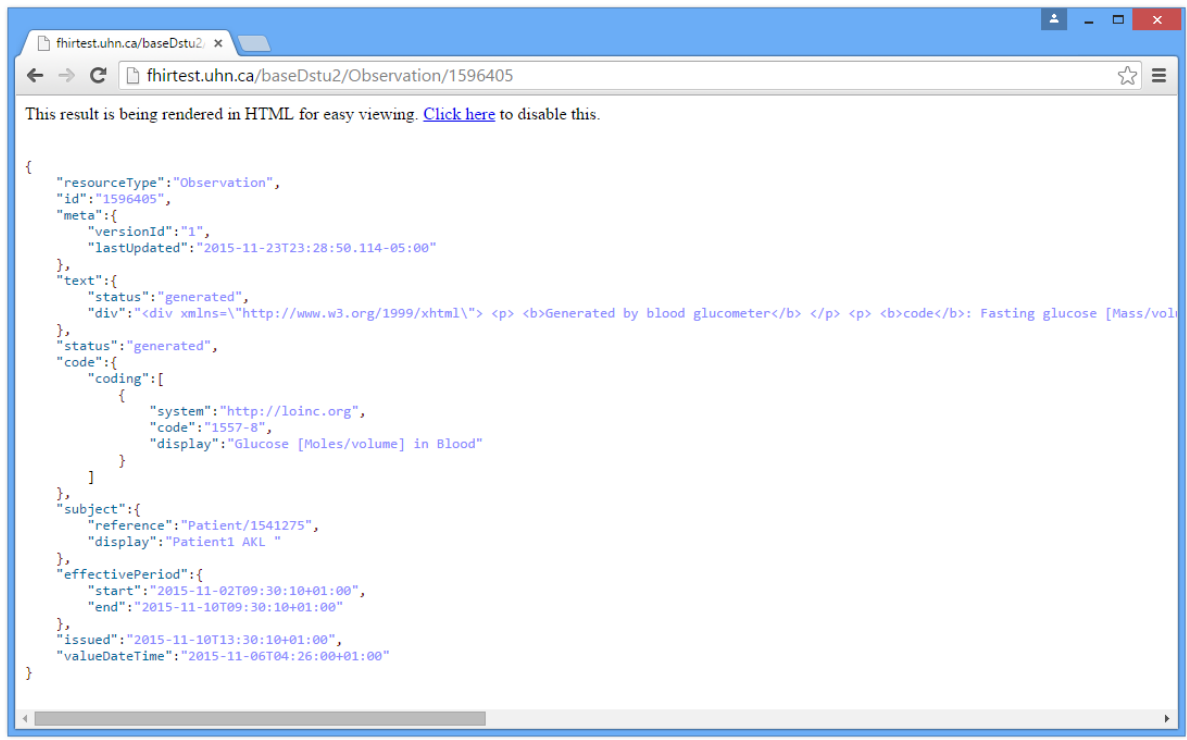

Figure 2: Uploaded blood glucose data with Observation resource 


\section{Related Projects Using FHIR}

Similar work has been done in the US using FHIR on an existing platform [32] which demonstrated that vendor applications could easily integrate the laboratory results of a patient. They adopted Meaningful Use Stage 2 [33] where a patient could access their health data from their health provider and achieve a better engagement with their provider. In another project [34], part of the architecture built was collecting weight from a weighing scale and integrating that data using FHIR resources.

\section{Discussion}

Blood glucose, blood pressure and BMI data were chosen as representing wellness data since they were related to two major chronic diseases, diabetes and hypertension. The different kinds of blood glucose readings depending on the meals and time, and different blood pressure readings in the mornings and evenings could be successfully stored in FHIR resources for testing the data model. Different patients may have different testing frequencies and upload the results to the clinicians/healthcare service providers in different time periods. The data model was sufficiently flexible to organize the data in these different scenarios.

The developed wellness data model had a hierarchical structure. The data model included a reference to the patient to whom the wellness data belonged which was a requirement to refer the patient while using FHIR to upload specific test data.

In a previous study by Jung at al. [35], after patients uploaded wellness data to the server of the healthcare provider, they got feedback on how to enhance their health. Consider such a scenario: a patient used to take three blood glucose readings per day and upload the data to the server weekly. After two months the doctor learns that his/her blood glucose is under control and suggests he/she upload data monthly and includes the highest, average and lowest readings for each week. In such a case the settings of the mobile app the patient uses can be easily adjusted and the doctor is free from possible information overload while maintaining a close watch on the patient. The data model is flexible to accept such change in the frequency of data readings.

It is also easy to reduce the types of data included in the data model since each list of data can be empty. For example a patient can upload only blood pressure data if his doctor does not need his blood glucose readings. On the other hand this wellness data is also easily extendible. In case the doctor wants to see the pulse rate or daily food intake of the patient, the model can include another list of pulse rate data or daily food intake data.

Any new resource should be built upon predefined data types and possibly base resources in FHIR. In the development of a new resource for wellness data, a key element is a reference to the resource "Patient" who owned the wellness data. This is done through the predefined complex type of "Reference".

The wellness data model could be extended to include more types of wellness data. The resource on FHIR could also be extended to accept the new wellness data by preparing new types of data. It demonstrated that FHIR was technically capable of expressing wellness data generated by patients. Thus the wellness data could be further integrated in EHR systems for better healthcare services.

A similar approach of organizing wellness data was followed by Open mHealth [36]. Open mHealth is based on 'common data schemas' which is the standardized data structure for each type of wellness data such as blood glucose, blood pressure and temperature [37]. For example the common data schema for blood pressure features similar information on effective time period, differentiating systolic and diastolic blood pressure.

The existing FHIR resources are not specifically designed for wellness data. However Observation, which is an existing official resource has the potential to express wellness data. Observation is a central element in healthcare and is used to support diagnosis, monitor progress and determine baselines and patterns. Observation records a data item and its related information about the performer, encounter, device, interpretation, status and so on.

Observation does not directly contain multiple values; the wellness data items which are organized in a single list in the new resource for wellness data have to be divided into individual data items. This leads to a large number of individual resource values in the FHIR server which could in turn result in more transactions of data exchange between the patient app and FHIR server. Fortunately FHIR provides a feature called "Bundle" [38] to better organize the uploading of multiple resources. A Bundle is a collection of resources which can be transferred to the server in a single instance. Different resources can be bundled together for operations such as creating, updating and deleting on a FHIR server as a single operation (transaction). The server will then respond with a complete set of affected resources. For example if a patient tests blood pressure both in the morning and evening, these two readings can then be uploaded to the server in two Observation resources combined in a single bundle. 
There were several public FHIR servers available for testing. Within this research project, the server at Hapi FHIR [39] was chosen for testing. It was supported by an open source project in Github. It supported all FHIR official resource types and operations. It also had a query builder UI which was useful to facilitate testing. There are also other public FHIR servers [40] which provide web based tools to help build resources and testing.

\section{Conclusion}

Wellness data and PHR data are important for healthcare service providers to offer better healthcare services. However these were not widely utilized for several reasons and one of the key issues was the lack of interoperability with EHR systems. To address interoperability issues, this research project aimed to evaluate the suitability of FHIR which is expected to be the next worldwide healthcare information exchange standard. A conceptual wellness data model was developed to include representable types of data chosen from a wide range of wellness data. It was mapped to an existing FHIR resource Observation to allow the exchange of wellness data between FHIR server and the patient. With some built-in techniques of FHIR it was possible to use Observation for wellness data integration.

\section{References}

[1] A. Etzioni, "Personal Health Records Why Good Ideas Sometimes Languish", Issues in Science and Technology, vol. 26(4), pp. 59-66, 2010.

[2] H. Li, J. Zhang, and R. Sarathy, "Examining the decision to use standalone personal health record systems as a trustenabled fair social contract", Decision Support Systems, vol. 57, pp. 376-386, 2014.

[3] M. J. Witry, W. R. Doucette, J. M. Daly, B. T. Levy and E. A. Chrischilles, "Family Physician Perceptions of Personal Health Records", Perspectives in Health Information management. American Health Information Management Association, 2010.

[4] J. M. Grossman, T. Zayas-Cabán, and N. Kemper, Information Gap: Can Health Insurer Personal Health Records Meet Patients' and Physicians' Needs?. Health Affairs, 28(2), pp 377-389, 2009.

[5] J. H. Hibbard and J. Greene, What the evidence shows about patient activation: better health outcomes and care experiences; fewer data on costs. Health affairs, 32(2), pp 207-214, 2013.

[6] J. D. Piette, M. Weinberger, F. B. Kraemer and S. J. McPhee, Impact of automated calls with nurse follow-up on diabetes treatment outcomes in a Department of Veterans Affairs Health Care System A Randomized Controlled Trial. Diabetes care, 24(2), pp 202-208, 2001.

[7] N. Archer, U. Fevrier-Thomas, C. Lokker, K. A. McKibbon and S. E. Straus, Personal health records: a scoping review. Journal of the American Medical Informatics Association, 18(4), pp 515-522, 2011.

[8] A. Darkins, P. Ryan, R. Kobb, L. Foster, E. Edmonson, B. Wakefiel and A. E. Lancaster, "Care Coordination/Home Telehealth: The Systematic Implementation of Health Informatics, Home Telehealth, and Disease Management to Support the Care of Veteran Patients with Chronic Conditions", Telemedicine and e-Health, vol. 14(10), pp. 1118-1126, 2008.

[9] HL7.org. (2016). Resource List - FHIR. Available: http://www.hl7.org/FHIR/resourcelist.html

[10] D. Bender and S. Kartipi, HL7 FHIR: An agile and

RESTful approach to healthcare information exchange. IEEE 26th International Symposium on Computer-Based Medical Systems (CBMS), pp 326-331, 2013.

[11] Institute of Medicine. (2012). Living Well with Chronic Illness: A Call for Public Health Action. Available: https://iom.nationalacademies.org/ /media/Files/Report\%20F iles/2012/Living-Well-with-Chronic-

Illness/livingwell_chronicillness_reportbrief.pdf

[12] M. E. Seddon, M. N. Marshall, S. M. Campbell and M. O. Roland, Systematic review of studies of quality of clinical care in general practice in the UK, Australia and New Zealand. Quality in health care, 10(3), pp 152-158, 2001.

[13] American Diabetes Association, Standards of medical care for patients with diabetes mellitus. Puerto Rico Health Sciences Journal, 20(2), 2013.

[14] World Health Organization (WHO. (2016). A global brief on hypertension: silent killer, global public health crisis. World, http://chronicconditions.thehealthwell.info/searchresults/global-brief-hypertension-silent-killer-global-publichealth-crisis, Accessed August 25, 2016.

[15] C. Quinn, S. Clough, J. Minor, D. Lender, M. Okafor and A. Gruber-Baldini, "WellDoc"TM mobile diabetes management randomized controlled trial: change in clinical and behavioral outcomes and patient and physician satisfaction", Diabetes Technology \& Therapeutics, vol. 10(3), pp. 160-168, 2008.

[16] P. Plastiras, D. O'Sullivan and P. Weller, An OntologyDriven Information Model for Interoperability of Personal and Electronic Health Records. In eTELEMED 2014, The Sixth International Conference on eHealth, Telemedicine, and Social Medicine, pp. 130-133, 2014.

[17] JASON, Mitre Corporation. A robust health data infrastructure. US Agency for Healthcare Research and Quality publication no. 14-0041-EF. http://healthit.gov/sites/ default/ files/ptp13-700hhs_white.pdf. Published April 2014. Accessed 21 August, 2016.

[18] S. Hull, Patient-Generated Health Data Foundation for Personalized Collaborative Care. Computers Informatics Nursing, 33(5), pp 177-180, 2015.

[19] E. Årsand, N. Tatara, G. Østengen and G. Hartvigsen, "Mobile phone-based self-management tools for type 2 diabetes", Journal of Diabetes Science and Technology, vol. 4(2), pp. 328-336, 2010.

[20] D. H. Frøisland, E. Årsand and F. Skårderud, "Improving diabetes care for young people with type 1 diabetes through visual learning on mobile phones: mixedmethods study", Journal of Medical Internet Research, vol. 14(4), pp. e111, 2012. 
[21] L. T. Harris, J. Tufano, T. Le, C. Rees, G. A. Lewis, A. B. Evert and J. D. Ralston, "Designing mobile support for glycemic control in patients with diabetes", Journal of biomedical informatics, vol. 43(5), pp. 37-40, 2010.

[22] R. S. Istepanian, K. Zitouni, D. Harry, N. Moutosammy, A. Sungoor, B. Tang and K. A. Earle, "Evaluation of a mobile phone telemonitoring system for glycaemic control in patients with diabetes", Journal of Telemedicine and Telecare, vol. 15(3), pp. 125-128, 2009.

[23] C. R. Lyles, L. T. Harris, T. Le, J. Flowers, J. Tufano, D. Britt and J. D. Ralston, "Qualitative evaluation of a mobile phone and web-based collaborative care intervention for patients with type 2 diabetes", Diabetes Technology \& Therapeutics, vol. 13(5), pp. 563-569, 2011.

[24] C. C. Quinn, M. D. Shardell, M. L. Terrin, E. A. Barr, S. H. Ballew and A. L. Gruber-Baldini, "Cluster-randomized trial of a mobile phone personalized behavioral intervention for blood glucose control", Diabetes Care, vol. 34(9), pp. 1934-1942, 2011.

[25] J. Lee, "EMR Management System for Patient Pulse Data", Journal of Medical Systems, vol. 36(5), pp. 32553260, 2012.

[26] M. Shapiro, D. Johnston, J. Wald and D. Mon, "Patientgenerated health data", RTI International, 2012.

[27] C. D. Saudek, R. L. Derr and R. R. Kalyani, “Assessing Glycemia in Diabetes Using Self-monitoring Blood Glucose and Hemoglobin A1c", Journal of the American Medical Association, vol. 295(14), pp. 1688-1697, 2006.

[28] G. Dailey, "Assessing Glycemic Control with Selfmonitoring of Blood Glucose and Hemoglobin A1c Measurements", Mayo Clinic Proceedings, vol. 82(2), pp. 229-236, 2007.

[29] Y. Imai, K. Kario, K. Shimada, Y. Kawano, N. Hasebe, H. Matsuura and M. Miyakawa, "The Japanese Society of Hypertension Guidelines for Self-monitoring of Blood Pressure at Home", Hypertension research: official journal of the Japanese Society of Hypertension, vol. 35(8), pp. 777, 2012.

[30] T. G. Pickering, Self-monitoring of blood pressure. Blood pressure monitoring in cardiovascular medicine and therapeutics, Humana Press, pp. 3-37, 2007.

[31] LOINC, http://loinc.org/, Accessed August 21, 2016.

[32] J. C. Mandel, D. A. Kreda, K. D. Mandl, I. S. Kohane, and R. B. Ramoni, SMART on FHIR: a standards-based, interoperable apps platform for electronic health records. Journal of the American Medical Informatics Association, ocv189, pp 899-908, 2016.

[33] Meaningful Use Stage 2, https://www.healthit.gov/providers-professionals/step-5achieve-meaningful-use-stage-2, Accessed August 28, 2016. [34] B. Franz, A. Schuler and O. Kraus, Applying FHIR in an Integrated Health Monitoring System. EJBI, 11(2), pp en51en56, 2015.

[35] E.Y. Jung, J. Kim, K.Y. Chung and D. K. Park, "Mobile healthcare application with EMR interoperability for diabetes patients", Cluster Computing, vol. 17(3), pp. 871-880, 2013. [36] Open mHealth. (2015). Blood pressure. Available: Open mHealth:http://www.openmhealth.org/documentation/\#/sche ma-docs/schema-library/schemas/omh_blood-pressure,

Accessed September 15, 2015.

[37] Open mHealth. (2015). Schema library. Available: Open mHealth:http://www.openmhealth.org/documentation/\#/sche ma-docs/schema-library, Accessed September 15, 2015.

[38] Bundle, https://www.hl7.org/fhir/bundle.html, Accessed September 30, 2015.

[39] Hapi FHIR, http://fhirtest.uhn.ca/, Accessed October 15, 2015.

[40] Clinfhir, http://clinfhir.com/, Accessed October 15,2015 . 